\title{
GOÄ - Wenn der Schwanz mit dem Hund wedelt
}

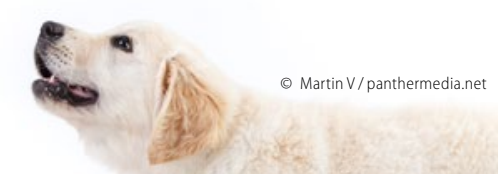

Liebe Kolleginnen und Kollegen,

bis vor Kurzem haben wir die Entwicklung der GOÄ-Novelle mit Spannung verfolgt. Schon 2011 wurde der Bundesärztekammer (BÄK) vom Bundesministerium für Gesundheit (BMG) ins Lastenheft der neuen GOÄ diktiert, sich zur Arbeitserleichterung mit dem Verband der privaten Krankenversicherer an einen Tisch zu setzen und eine gemeinsame Vorlage zu entwickeln. Die Beihilfe könnte sich dann an dieses Konstrukt anhängen, wenn sie sich das leisten wollte, so dachte man sich. Die Preise würden damit also von Erstattungsstellen diktiert werden. Das wäre wohl einmalig. Diese ganze Aktion sollte zudem hinter verschlossenen Türen stattfinden, um die Ärzteschaft nicht unnötig aufzuwühlen.

Man hatte die Rechnung allerdings ohne die denkenden organisierten Teile der Ärzteschaft gemacht. Es wurden Verhandlungsergebnisse publik, obwohl Stillschweigen zwischen den Parteien vereinbart war. Zum Beispiel hat ein Verhandlungsführer der Ärzteschaft in der Zwischenzeit das Schiff gewechselt und arbeitet nicht mehr als Hauptgeschäftsführer bei der Bundesärztekammer, sondern als Verwaltungsdirektor bei der Kassenärztlichen Bundesvereinigung. Da wundert es wenig, wenn die GOÄ sehr stark den Charakter des Einheitlichen Bewertungsmaßstabs (EBM) bekam, mit allem möglichen Ungemach. Etwa mit der neuen Systematik eines „robusten Einfachsatzes", der nur noch mit Einverständnis eines eigens dafür geschaffenen Gremiums, der Gemeinsamen Kommission (GeKo), gesteigert werden darf.

\section{Therapiefreiheit ade}

Die GeKo ist paritätisch besetzt mit Ärzten auf der einen Seite und PKV und Beihilfe auf der anderen Seite. Die Ärzte sind alle zwei Jahre alternierend als BÄK- und KBV-Vertreter eingebunden. Aber dieser "Private Gemeinsame Bundesausschuss" hat noch weitere Aufgaben. Er soll die Angemessenheit der Honorarentwicklung mithilfe einer noch zu schaffenden (und natürlich auch von den Ärzten zu finanzierenden) Datensammelstelle innerhalb von 36 Monaten nach Inkrafttreten der GOÄ evaluieren.

Dass künftig nicht mehr die beste medizinische Versorgung im Vordergrund und im Paragrafenteil festgeschrieben steht, sondern die wirtschaftlichste, davon haben die privaten Versicherer sicher schon lange geträumt. Dass endlich Schluss ist mit den vielen analogen Berechnungen, entlastet die Sachbearbeiter. Und mit Analogberechnungen ist auch weitgehend Schluss: Die Ärzte sollen eine Stichtagsregelung bekommen, wie wir sie in der GOZ 1988 kennen und hassen gelernt haben.

Klar auch, dass wieder stärker in Komplexleistungen gedacht wird und das sogenannte Zielleistungsprinzip zum Tragen

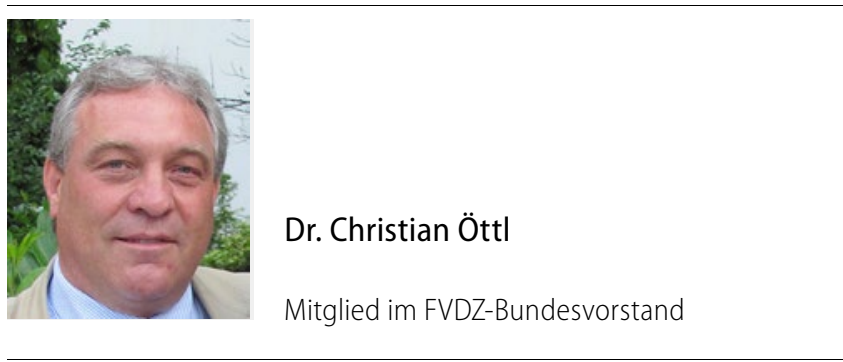

kommt. Nach dem Motto: „Alles schon in der Hauptleistung inklusive!“.

Das Schmankerl zum Schluss: Medizinische Versorgungszentren und Krankenhausambulanzen müssen sich nicht an die GOÄ halten, „werden es aber aus Praktikabilitätsgründen wohl machen", lauten Mutmaßungen. Ein Schelm, wer dabei an eine Öffnungsklausel denkt. Zusätzlich dürfen nämlich von der großen GeKo auch „Modellvorhaben zur Verbesserung der Versorgung“ auf den Weg gebracht werden - „Öffnungsklausel 2.0“. Klar auch, dass zur Erbringung einer Leistung die fachliche Qualifikation nach Maßgabe des Weiterbildungsrechts nachgewiesen werden muss; natürlich nicht, um Geld zu sparen - alles nur Patientenschutz. Therapiefreiheit ade.

\section{Weg in die Bürgerversicherung}

Und die Rechnungsstellung bleibt natürlich auch nicht ungeschoren. Nicht nur muss innerhalb von sechs Monaten eine Rechnung erstellt sein, sie muss künftig maschinenlesbar sein. Der Arzt wird faktisch in das Erstattungsgebaren der Versicherung eingebunden, indem er über jede denkbare Nichterstattung aufzuklären hat. Vieles aus der missratenen „SchmidtGOZ", die die Zahnärzteschaft durch geschlossenes Auftreten verhindern konnte - steht jetzt bei den Ärzten im Entwurf. Um diesen Entwurf durchzudrücken, behaupten BÄK-Präsident Frank Ulrich Montgomery und der SPD-Gesundheitsexperte Karl Lauterbach, die Alternative wäre die Bürgerversicherung. Fakt ist aber, dass diese GOÄ den Weg in die Bürgerversicherung ebnet.

Diese GOÄ muss wegen der „Tschernobyl-Wirkung“, also Strahlung mit Langzeitwirkung, verhindert werden.

\section{Meint Ihr}
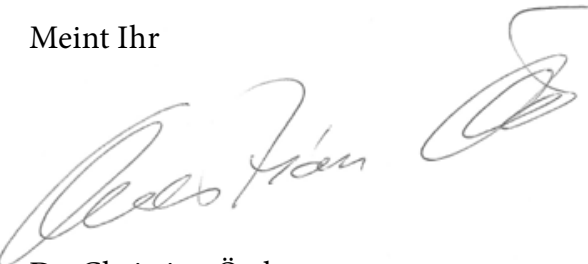

Dr. Christian Öttl 